\title{
Role of Structural Vacancies in the Stabilization of the Basic B1 Structure in Nonstoichiometric Titanium Monoxide $\mathrm{TiO}_{y}$
}

\author{
M. G. Kostenko ${ }^{a}$, A. V. Lukoyanov ${ }^{b, c}$, V. P. Zhukov ${ }^{a}$, A. A. Valeeva ${ }^{a, c}$, and A. A. Rempel ${ }^{a, c}$ \\ ${ }^{a}$ Institute of Solid State Chemistry, Ural Branch, Russian Academy of Sciences, \\ ul. Pervomaiskaya 91, Yekaterinburg, 620990 Russia \\ ${ }^{b}$ Institute of Metal Physics, Ural Branch, Russian Academy of Sciences, \\ ul. S. Kovalevskoi 18, Yekaterinburg, 620990 Russia \\ ${ }^{c}$ Yeltsin Ural Federal University, ul. Mira 19, Yekaterinburg, 620002 Russia
}

\begin{abstract}
The electron structures and formation enthalpies of vacancy-free cubic TiO, vacancy-ordered monoclinic $\mathrm{Ti}_{5} \mathrm{O}_{5}$, and vacancy-disordered cubic $\mathrm{TiO}_{y}$ have been investigated using DFT+GGA calculations. $\mathrm{Ti}_{5} \mathrm{O}_{5}$ was found to be the stablest phase and $\mathrm{TiO}$ was found to be the least stable. The reason for the stability of the titanium and oxygen vacancies in the basic $B 1$ structures of $\mathrm{Ti}_{5} \mathrm{O}_{5}$ and $\mathrm{TiO}_{y}$ has been deduced. The titanium vacancies lead to a decrease in the Fermi energy. Oxygen vacancies cause strengthening in covalent $\mathrm{Ti}-\mathrm{Ti}$ bonding.
\end{abstract}

DOI: $10.3103 / \mathrm{S} 1062873813030210$

Titanium monoxide $\mathrm{TiO}_{y}$ is one of the few nonstoichiometric compounds with a high concentration of structural vacancies in both sublattices. Their percentage can be up to 32 at $\%[1-4]$. In the case of an equiatomic composition, the amount of vacancies is about $14-15$ at $\%$ in each sublattice $[3,4]$. When the composition deviates from stoichiometric, the concentration of vacant sites increases in one sublattice and decreases in another [1]. The high content of vacancies results in a wide homogeneity of titanium monoxide, which is in the range of $y=0.7-1.25$ [3].

Three stoichiometric crystal modifications of titanium monoxide composition are known. They all have the basic $B 1$ structure, but they differ from each other by the presence of vacancies and their specific location. One can obtain a phase without structural vacancies by annealing at high pressures [5]. At low pressures, the thermodynamic equilibrium phases are those with vacancies. Vacancies may be located over sites of the basic $B 1$ structure either randomly or in an ordered manner. The disordered state is thermodynamically stable at temperatures above $1300 \mathrm{~K}$, but it can be obtained at room temperature by quenching $[3,4]$. Since the vacancies are chaotically located, the $X$-ray diffraction patterns of disordered samples exhibit only characteristic of the cubic $B 1$ structure. The vacancies are ordered during slow annealing from the temperature of the order-disorder phase transition; the crystal symmetry is lowered to monoclinic, so a $\mathrm{Ti}_{5} \mathrm{O}_{5}$ phase is formed $[3,4]$.

Thus, the presence of vacancies and their ordering is energetically favorable for titanium monoxide. However, the role of vacancies in stabilization of the basic $B 1$ structure is poorly understood. There are many theoretical studies dedicated to this problem [6-13]. For example, the electron structure of the TiO and $\mathrm{Ti}_{5} \mathrm{O}_{5}$ phase and disordered stoichiometric $\mathrm{TiO}_{1.0}$ phase have been calculated in [12]. However, the ordered and disordered phases have been studied by various methods, which has made it impossible to compare their total energies adequately. In [13], only $\mathrm{TiO}$ and $\mathrm{Ti}_{5} \mathrm{O}_{5}$ were considered. It was shown that a crucial role in stabilizing the ordered phase is played by oxygen vacancies; however, the reasons for the stability of the disordered state have not been considered. Thus, what the role of metal and nonmetal vacancies in disordered and ordered phase are remains an open question.

This paper is aimed at determining the contribution of titanium and oxygen vacancies in the stabilization of the basic $B 1$ structure and to investigate the stability of crystal titanium monoxide modifications. To do this, we calculated the electron structures and total energies of the three phases: vacancy-free cubic TiO, vacancy-ordered monoclinic $\mathrm{Ti}_{5} \mathrm{O}_{5}$, and vacancy-disordered cubic $\mathrm{TiO}_{y}$; analyzed the features of the chemical bond; and found the stablest crystal modification.

The electron structure was calculated with the density functional theory and account for the exchangecorrelation potential in the approximation of the generalized gradient correction. The PWSCF code of the Quantum ESPRESSO software suite was used for the calculations, which assumes the use of pseudopotentials [14]. The appearance of vacancies in the structure may cause displacement of atoms relative to the basic structure, so we relaxed the atomic positions. 
$N(E), \mathrm{eV}^{-1}$ supercell ${ }^{-1}$

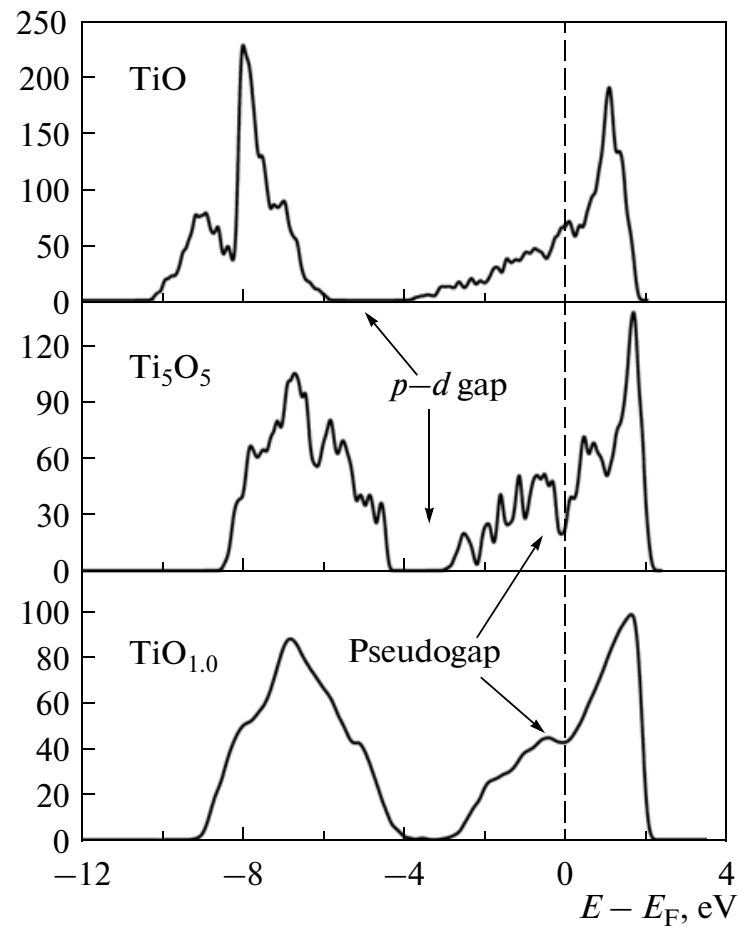

Fig. 1. Density of electron states of titanium monoxide per supercell: (a) vacancy-free phase $\mathrm{TiO}$, (b) monoclinic $\mathrm{Ti}_{5} \mathrm{O}_{5}$ phase, and (c) disordered stoichiometric $\mathrm{TiO}_{1.0}$ phase. Plots are combined by the Fermi energy.

When studying the electron structure of the disordered titanium monoxide, the main problem is how to simulate disorder in the structure. Only the coherent potential approximation has been used up to now. However, this approach cannot account for static atom displacements and does not provide exact values of the total energy. In this paper, we used a supercell method. A supercell was formed by twofold translation of the unit cell of the monoclinic phase [4] along the $a$ and $b$ crystallographic directions. Since there is insufficient experimental data on the existence of short-range order in $\mathrm{TiO}$, vacancies in the supercell were distributed randomly. This approach makes it possible to use, instead of coherent potentials, the potentials of real elements, as well as compare the energy parameters of the lattices of the ordered and disordered phases.

The calculation revealed the electron spectrum peculiarities of three types of titanium monoxide (Fig. 1). All three phases are metals. However, in the case of the vacancy-ordered state, a pseudogap arises at the Fermi level, which significantly decreases under disordering. The bandwidth of the occupied states is reduced by about $1 \mathrm{eV}$ with the appearance of vacancies in the basic structure. This is agrees with the experimental data [15]. The low-energy part of the band of occupied states mainly includes $2 p$ states of oxygen, and the high-energy part includes $3 d$ states of (a)

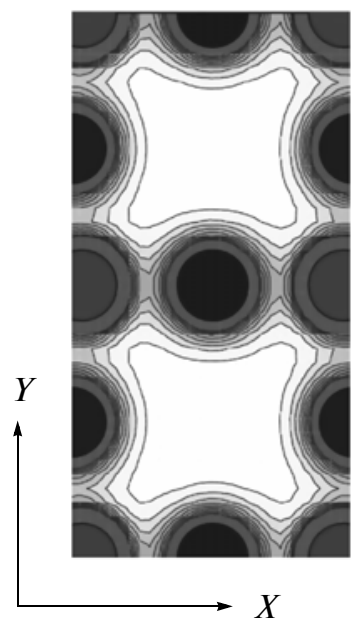

(b)

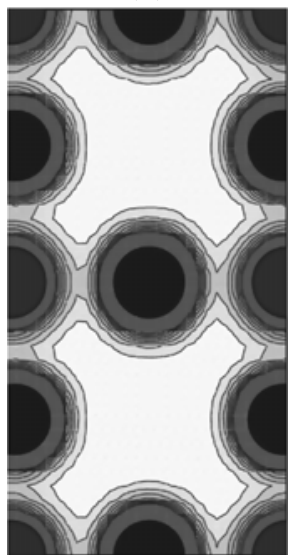

Fig. 2. Electron density map of (a) titanium sublattice vacancy channel and (b) oxygen sublattice vacancy channel of the ordered monoclinic $\mathrm{Ti}_{5} \mathrm{O}_{5}$ phase in the $X Y$ plane.

titanium. In the case of the vacancy-free and ordered phase, the $p-d$ gap is clearly pronounced; in disordering, it is replaced by a negligible but nonzero density of states.

The electron density map (Fig. 2) suggests that the charge density of oxygen vacancies is higher than that of titanium ones. The charge redistribution between the two types of vacancies, first, partially compensates the Madelung energy loss due to vacancy formation, and, second, indicates the occurrence of new chemical bonds.

An electron structure study of the disordered titanium monoxide, depending on the composition (Fig. 3), showed that the formation of the pseudogap is due to the presence of vacancies in the oxygen sublattice. Without vacancies in the sublattice, the pseudogap disappears completely. In contrast, the value of the Fermi energy correlates well with the fracture of vacancies in the titanium sublattice. The Fermi energy is minimum at the upper border of the homo- 
geneity region, when the number of vacancies reaches $25 \%$ in the titanium sublattice (Table 1 ). Thus, the role of vacancies in the oxygen sublattice consists in the formation of bonds between titanium atoms, which leads to a redistribution of states below the Fermi level and occurrence of the pseudogap. The role of vacancies in the titanium sublattice is to reduce the energy of electrons.

Analysis of the partial densities of electron states has shown that if vacancies are completely absent, two of the five $3 d$ orbitals of titanium are involved in bonding with $2 p$ orbitals of oxygen, and the other three form bonds with titanium atoms, dictating the metal properties of the compound. However, if in the perfect $B 1$ structure all the titanium and oxygen atoms are indistinguishable from each other, then in the structure with vacancies, the atoms of each sublattice are divided into groups depending on the type of nearest environment of the atom. There are an infinite number of such types in the disordered phase. In the ordered phase, each sublattice can include three types, depending on the number of vacancies in the first, second, and third coordination spheres: $X_{2 / 2}^{0}, X_{1 / 3}^{0}$, and $X_{1 / 2}^{2}$. where $X$ is a chemical element. A left subscript indicates the number of vacancies in the first coordination sphere; the right subscript, the number of vacancies in the second coordination sphere; and the superscript shows the number of vacancies in the third coordination sphere. The vacancies in the ordered phase are arranged to form vacancy channels in the titanium and oxygen sublattices [3, 4].

For titanium atoms located in the vacancy channel of oxygen sublattice $\left(\mathrm{Ti}_{2 / 2}^{0}\right)$, there are significant changes in the energy and the occupancy of an $x^{2}-y^{2}$ orbital. This suggests new links between titanium atoms through the vacant oxygen site. Furthermore, covalent bonds are strengthened between the titanium atoms surrounding the oxygen vacancy. The above facts lead to the occurrence of the pseudogap at the Fermi level. The vacancy channels are destroyed in disordering and the pseudogap at the Fermi level virtually disappears.

The results of calculation of the formation enthalpy are listed in Table 2. The ordered phase is the most energetically favorable. The stability of the disordered state is decreased; however, the least stable is the phase with a complete lack of vacancies. The calculated enthalpy values are in a good agreement with the experimental data [16].

Thus, the formation of vacancies and their ordering in titanium monoxide stabilizes the basic $B 1$ structure. The ordered phase is stabler, because it contains vacancy channels. The structure and location of these channels gives the phase monoclinic symmetry and explains why the vacancy rate is just $1 / 6$. This concen-
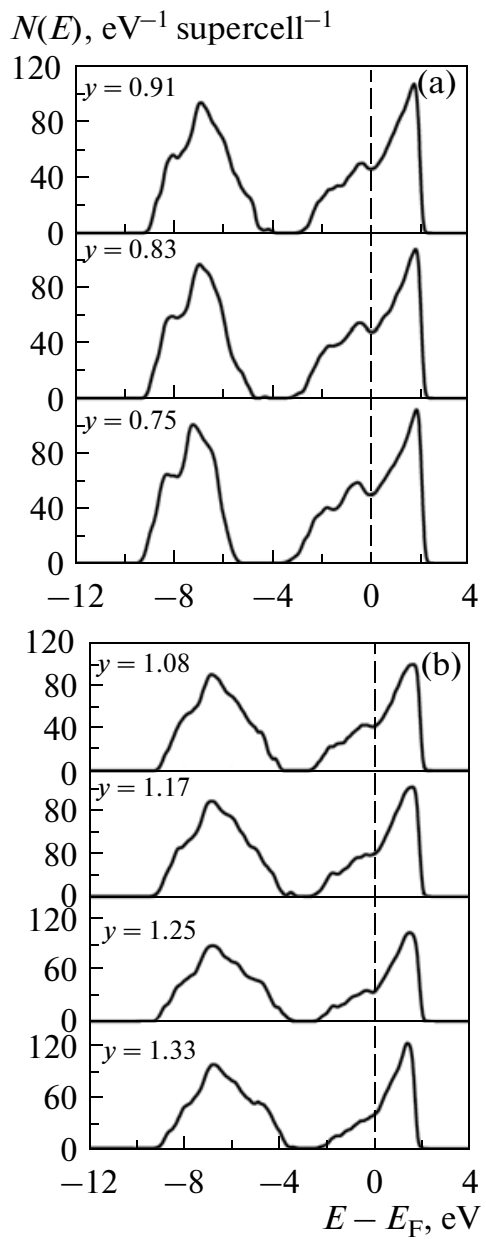

Fig. 3. Densities of electron states of the disordered titanium $\mathrm{TiO}_{y}$ monoxide for (a) $y<1.0$ and (b) $y>1.0$, calculated with a step of $1 / 12$ along $y$. Plots are combined by the Fermi energy.

tration corresponds to the maximum possible number of channels in which neighboring vacancies are sufficiently distant from each other in the structure. The vacancies in the titanium sublattice reduce the Fermi energy substantially. Their arrangement on the same

Table 1. Fracture of atomic positions $x$ in the titanium sublattice and $z$ in the oxygen sublattice of the disordered titanium monoxide and calculated values of the Fermi energy $E_{\mathrm{F}}$

\begin{tabular}{l|c|c||c|c|c}
\hline$x$ & $z$ & $E_{\mathrm{F}}, \mathrm{eV}$ & $x$ & $z$ & $E_{\mathrm{F}}, \mathrm{eV}$ \\
\hline 1 & 0.75 & 16.68 & 0.81 & 0.88 & 14.42 \\
0.94 & 0.78 & 15.80 & 0.79 & 0.92 & 14.36 \\
0.89 & 0.81 & 15.04 & 0.77 & 0.96 & 14.28 \\
0.83 & 0.83 & 14.44 & 0.75 & 1 & 13.85 \\
\hline
\end{tabular}


Table 2. Calculated and experimental values of formation enthalpy $\Delta H$ of monoxide titanium: vacancy-free phase $\mathrm{TiO}$, monoclinic $\mathrm{Ti}_{5} \mathrm{O}_{5}$ phase, and disordered phase of stoichiometric composition $\mathrm{TiO}_{1.0}$

\begin{tabular}{l|c|c}
\hline \multicolumn{1}{c|}{ Phase } & $\begin{array}{c}\Delta H, \mathrm{~kJ} \mathrm{~mol}^{-1}, \\
\text { theory }\end{array}$ & $\begin{array}{c}\Delta H, \mathrm{~kJ} \mathrm{~mol}^{-1}, \\
\text { experiment }\end{array}$ \\
\hline $\mathrm{TiO}$ & -498.0 & - \\
$\mathrm{Ti}_{5} \mathrm{O}_{5}$ & -547.6 & - \\
$\mathrm{TiO}_{1.0}$ & -521.8 & $-518.4[16]$ \\
\hline
\end{tabular}

type with oxygen vacancies partly compensates Madelung energy losses in the formation of vacancies.

\section{ACKNOWLEDGMENTS}

This study was supported by the Russian Foundation for Basic Research, project no. 13-03-00164; and by the Ural Branch of Russian Academy of Sciences, project no. 12-M-23-2001 and 13-3-NP-532.

\section{REFERENCES}

1. Valeeva, A.A., Rempel, A.A., Sprengel, W., and Schaefer, H.-E., Phys. Rev. B, 2007, vol. 75, p. 094107 1.

2. Gusev, A.I., Rempel, A.A., and Magerl, A., Disorder and Order in Strongly Non-Stoichiometric Compounds. Transition Metal Carbides, Nitrides and Oxides, Berlin: Springer, 2001.
3. Watanabe, D., Terasaki, O., Jostsons, A., and Castles, J.R., in The Chemistry of Extended Defects in Non-Metallic Solids, Eyring, L. and O'Keefe, M., Eds., AmsterdamLondon: North-Holland, 1970, p. 238.

4. Valeeva, A.A., Rempel', A.A., and Gusev, A.A., Neorg. Mater., 2001, vol. 37, no. 6, p. 603.

5. Taylor, A. and Doyle, N.J., High Temp. - High Press., 1969, vol. 1, p. 679.

6. Goodenough, J.B., Phys. Rev. B, 1972, vol. 5, p. 2764.

7. Huisman, L.M., Carlsson, A.E., Gelatt, C.D., Jr., and Ehrenreich, H., Phys. Rev. B, 1980, vol. 22, p. 991.

8. Gubanov, V.A., Ivanovsky, A.L., Shveikin, G.P., and Ellis, D.E., J. Phys. Chem. Solids, 1985, vol. 46, p. 383.

9. Hörmandinger, G., Redinger, J., Weinberger, P., et al., Solid State Commun., 1988, vol. 68, p. 467.

10. Schlapansky, F., Herzig, P., Eibler, R., Hobiger, G., and Neckel, A., Z. Phys. B: Condens. Matter, 1989, vol. 75, p. 187.

11. Barman, S.R. and Sarma, D.D., Phys. Rev. B, 1994, vol. 49, p. 16141.

12. Andersson, D.A., Korzhavyi, P.A., and Johansson, B., Phys. Rev. B, 2005, vol. 71, p. 144101-1.

13. Graciani, J., Marques, A., and Sang, J.F., Phys. Rev. B, 2005, vol. 72, p. 054117-1.

14. Giannozzi, P., Baroni, S., Bonini, N., et al., J. Phys. Condens. Matter, 2009, vol. 21, p. 395502.

15. Bartkowski, S., Neumann, M., Kurmaev, E.Z., et al., Phys. Rev. B, 1997, vol. 56, p. 10656.

16. Humprey, G.L., J. Am. Chem. Soc., 1951, vol. 73, p. 1587. 